Article

\title{
Antimicrobial Resistance of Coagulase-Positive Staphylococcus Isolates Recovered in a Veterinary University Hospital
}

\author{
Marta Pérez-Sancho ${ }^{1,2, *}$, Sergio Alvarez-Perez ${ }^{1,3}(0)$, Teresa Garcia-Seco ${ }^{2} \oplus$, Marta Hernandez ${ }^{4}$, \\ David Rodríguez-Lázaro ${ }^{5}$ (D) , Lucas Domínguez ${ }^{1,2}$, Marta Eulalia García ${ }^{1,3}$ and \\ Jose Luis Blanco ${ }^{1,3}$ (D) \\ 1 Department of Animal Health, Faculty of Veterinary Medicine, Complutense University of Madrid, \\ 28040 Madrid, Spain; sergioaperez@ucm.es (S.A.-P.); lucasdo@ucm.es (L.D.); \\ megarcia@vet.ucm.es (M.E.G.); jlblanco@ucm.es (J.L.B.) \\ 2 VISAVET Health Surveillance Centre. Complutense University, 28040 Madrid, Spain; \\ teresagsr@visavet.ucm.es \\ 3 Veterinary Teaching Hospital, Faculty of Veterinary Medicine, Complutense University, 28040 Madrid, Spain \\ 4 Laboratorio de Biología Molecular y Microbiología, Instituto Tecnológico Agrario de Castilla y León, \\ 47071 Valladolid, Spain; ita-HerPerMa@itacyl.es \\ 5 Área de Microbiología, Departamento de Biotecnología y Ciencia de los Alimentos, Universidad de Burgos, \\ 09001 Burgos, Spain; drlazaro@ubu.es \\ * Correspondence: maperezs@ucm.es; Tel.: +34-913-944-096
}

Received: 30 September 2020; Accepted: 28 October 2020; Published: 29 October 2020

check for updates

\begin{abstract}
The Staphylococcus pseudintermedius group (SIG) is an emerging threat in veterinary medicine, particularly methicillin-resistant (MRSP) isolates, which are frequently associated with multidrug resistance. Reliable identification of SIG members is critical to establish correct antimicrobial treatments. However, information on the molecular epidemiology and antimicrobial resistance patterns of MRSP in some regions is still limited. This study aimed to assess the antimicrobial resistance of SIG isolates recovered from animals at the Veterinary Teaching Hospital of Complutense University of Madrid (Spain) during a 10-year period (2007-2016). A total of 139 selected Staphylococcus isolates were subjected to species-level identification by different bioanalytical techniques (PCR, VITEK, MALDI-TOF) and subsequent antimicrobial susceptibility testing. Methicillin-resistant isolates $(n=20)$ were subjected to whole genome sequencing for further characterization of their antibiotic resistance determinants. Our results showed that there was a good correlation between PCR and MALDI-TOF identification, whereas VITEK showed very divergent results, thus confirming MALDI-TOF as a good alternative for species-level identification of coagulase-positive staphylococci. Notably, S. pseudintermedius, including the epidemic MRSP genotype ST71, was the only SIG species found among canine isolates. In addition, we found a high prevalence of multidrug resistance and resistance to fluoroquinolones, cephalosporins and macrolides. Finally, diverse genes associated with antibiotic resistance were detected among MRSP isolates, although the genetic basis of some of the resistant phenotypes (particularly to fluoroquinolones) could not be determined. In conclusion, our study reveals the circulation of MRSP in the veterinary setting in Spain, thus highlighting the emerging threat posed by this bacterial group and the need for further epidemiological surveillance.
\end{abstract}

Keywords: SIG group; MALDI-TOF; whole genome sequencing; antimicrobial resistance 


\section{Introduction}

Staphylococcus aureus is a well-recognized pathogen that is responsible for a plethora of clinical manifestations (from skin infections to endocarditis, including fatal outcomes) in humans and animals worldwide. Coagulase-positive Staphylococcus (CoPS) isolates other than S. aureus, including the members of the Staphylococcus intermedius group (SIG, namely, S. intermedius, S. pseudintermedius and $S$. delphini) are emerging as a serious threat in veterinary medicine, especially in small animals. In particular, S. pseudintermedius is now considered the main CoPS species recovered from household pets [1]. S. pseudintermedius is also part of the normal microbiome of small animals and is considered an opportunistic pathogen that is implicated in a variety of infections (skin, uterus, urinary tract, abscesses, etc.) [2]. Additionally, S. pseudintermedius may pose a serious but often neglected zoonotic risk [3].

Methicillin-resistant $S$. aureus (MRSA) strains have become a major cause of concern in animal and human medicine. Similarly, decreased antimicrobial susceptibility has been detected in S. pseudintermedius isolates from companion animals. Strains carrying methicillin resistance genes (methicillin-resistant Staphylococcus pseudintermedius, MRSP) are of special importance in animal and human pathology [1]. Detailed molecular characterization of dominant MRSP strains may increase our knowledge about their distribution, epidemiology, pathogenesis and potential transmission in a given region. For example, certain multi-locus sequence typing (MLST) clonal complexes of MRSP have characteristic antimicrobial resistance profiles [4]. MRSP often display multidrug resistance to different antibiotics used in veterinary applications [1]. This may pose a challenge for veterinarians due to the scarcity of alternative antibiotics that are suitable for the treatment of MSRP infections [4]. In addition, knowledge about the carriage of antimicrobial resistance marker genes by MRSP isolates provides insights into the genetic basis of phenotypic resistance and may lead to the optimal selection of antibiotics for therapy.

Correct species-level identification of the staphylococci is a critical step for the establishment of adequate antimicrobial treatments, and is key for successful clinical outcomes. In this sense, oxacillin minimum inhibitory concentration (MIC) breakpoints depend on the Staphylococcus species [5], which emphasizes the relevance of reliable species identification. In particular, the correct discrimination of S. intermedius and S. pseudintermedius is essential to clarify important aspects of their epidemiology [3]. Currently, there are no commercial rapid systems available for CoPS differentiation, which hinders their correct species-level identification in most diagnostic laboratories. In addition, different CoPS species cannot be distinguished by unique biochemical traits. Although molecular-based identification of CoPS isolates has been proposed using different molecular targets [6,7], including these methods in routine laboratory testing is highly demanding with regard to time and cost [5].

In recent years, the clinical relevance, molecular epidemiology and antimicrobial resistance patterns of MRSP have been assessed in different countries [8,9]. However, to our knowledge, no systematic study dealing with these issues has been carried out in Spain in domestic animals, and the scarce data currently available refers to healthy dogs [10-12]. Thus, the present study aimed to assess the prevalence and characteristics of $S$. pseudintermedius isolates recovered from animals at the Veterinary Teaching Hospital of Complutense University in Madrid (Madrid, Spain) over a period of ten years (2007-2016). Isolates were identified to the species level, and phenotypically and genotypically characterized.

\section{Results}

\subsection{Origin and Identification of Coagulase-Positive Staphylococcus Isolates}

Most Staphylococcus isolates analyzed in this study were recovered from dogs $(128 / 139,92.1 \%$ of the total), but isolates also came from other sources including horses $(n=4,2.9 \%)$, cats and mice $(n=2,1.4 \%$ each), and lion, sheep and swine $(n=1,0.7 \%$ each). Isolates were collected from ear exudates $(n=58,41.7 \%$ ), body fluids (urine, exudates from different origins, $n=38,27.3 \%$ ) or other 
origins (nails, hair, abscesses and other samples, $n=43,31 \%$ ). The results of the different CoPS identification techniques used in this study are summarized in Table 1. There was a good agreement for S. pseudintermedius ( $\mathrm{n}=117$ ) identification by PCR and MALDI-TOF $(\mathrm{k}=0.528)$. In contrast, concordance between PCR and VITEK was much lower $(\kappa=0.010)$. Considering MALDI-TOF as the gold standard technique for S. aureus identification, VITEK identified correctly all isolates but one, which was assigned to $S$. intermedius. However, VITEK misidentified 13 S. pseudintermedius and one S. schleiferi isolates as $S$. aureus. All three S. schleferi isolates were identified by PCR and MALDI-TOF. In general, the correlation between PCR and MALDI-TOF results was good while VITEK showed very divergent results.

Table 1. Comparison of results of the species-level identification of coagulase-positive Staphylococcus (CoPS) isolates $(n=139)$ yielded by multiplex species-specific PCR, VITEK and MALDI-TOF.

\begin{tabular}{cccc}
\hline Species & PCR $^{\text {a }}$ & VITEK & MALDI-TOF $^{\mathbf{b}}$ \\
\hline Staphylococcus aureus & NA $^{\mathrm{c}}$ & 32 & 19 \\
\hline Staphylococcus pseudintermedius & 117 & 19 & 112 \\
\hline Staphylococcus intermedius & 0 & 88 & 4 \\
\hline Staphylococcus schleiferi & 3 & 0 & 3 \\
\hline
\end{tabular}

a Sasaki et al., 2010 (based on partial nuc gene to differentiate among Staphylococcus intermedius group (SIG) members) performed as multiplex PCR systems. This PCR was considered the gold standard technique for SIG identification in the present study. ${ }^{\mathrm{b}}$ One isolate was identified as Staphylococcus spp. ${ }^{\mathrm{c}}$ Not applicable, identification of S. aureus was performed by VITEK and MALDI-TOF. MALDI-TOF was considered the gold standard technique for identification of this species.

\subsection{Antimicrobial Resistance Profiles}

The results of the antimicrobial susceptibility test are shown in Table 2. Almost all CoPS isolates $(131 / 139,94.24 \%)$ were resistant to at least one of the antimicrobials tested. Notably, 50 different antimicrobial resistance profiles were detected. While $33 \mathrm{CoP}$ isolates (24 S. pseudintermedius and 9 S. aureus) showed resistance exclusively to penicillin, the most frequent combination of antimicrobial resistance was tetracycline/penicillin ( $\mathrm{n}=19$, all S. pseudintermedius), and 66 isolates (47.48\%, 60 S. pseudintermedius and 6 S. aureus) were multidrug resistant (MDR), i.e. resistant to at least three distinct classes of antimicrobial agents. The trend in the proportion of AMR-CoPS isolates over time was assessed by comparing the number of resistant isolates at three different periods covering a similar number of isolates: 2007-2010 $(\mathrm{n}=44), 2011-2014(\mathrm{n}=44)$ and 2015-2016 $(\mathrm{n}=51)$. Among all of the tested antimicrobial agents, ciprofloxacin $(P=0.013)$, norfloxacin $(P=0.02)$, ofloxacin $(P=0.013)$ and trimethoprim-sulfamethoxazole $(P=0.029)$ showed increasing resistance rates over time (Cochran-Armitage Test) (Table 3).

The association between AMR and the clinical origin of the isolates (fluids, ear exudates or other sources) was not significant $(P>0.05$, multiple proportions Z-test), except for gentamicin $(P<0.05)$, for which $27.6 \%$ isolates from ear exudates but only $10.5 \%$ of isolates from body fluids were resistant to this antibiotic.

The three S. schleiferi isolates were susceptible to all antimicrobials except one isolate (strain 21S), which was resistant to fluoroquinolones (ciprofloxacin, ofloxacin and norfloxacin). S. pseudintermedius showed significantly higher resistance rates to kanamycin $(P=0.006)$, tetracycline $(P=0.001)$, trimethoprim-sulfamethoxazole $(P=0.003)$, clindamycin $(P=0.04)$ and chloramphenicol $(P=0.013)$ than $S$. aureus. However, $S$. aureus isolates were significantly more resistant to amikacin $(P=0.009)$ than $S$. pseudintermedius. 
Table 2. Resistance frequency of 19 S. aureus and 117 S. pseudintermedius isolates recovered at the Complutense Veterinary Teaching Hospital (Spain) between 2006 and 2017.

\begin{tabular}{|c|c|c|c|c|c|}
\hline \multirow{2}{*}{ Antibiotic Class } & \multirow{2}{*}{$\begin{array}{c}\text { Antibiotic } \\
\text { (Abbreviation) }\end{array}$} & \multirow{2}{*}{ Disk Content $(\mu)$} & \multirow{2}{*}{ Breakpoint } & \multicolumn{2}{|c|}{ Number of Resistant (\% Resistance) } \\
\hline & & & & S. aureus $(\mathrm{n}=19)$ & S. pseudintermedius $(\mathrm{n}=117)$ \\
\hline \multirow[t]{3}{*}{ Fluoroquinolones } & Ciprofloxacin $(C I P)^{a}$ & 5 & $\leq 15$ & $3(15.79)$ & $23(19.65)$ \\
\hline & Ofloxacin $(O F X)^{a}$ & 5 & $\leq 14$ & $4(21.05)$ & $22(18.80)$ \\
\hline & Norfloxacin (NOR) ${ }^{a}$ & 10 & $\leq 12$ & $5(26.31)$ & $22(18.80)$ \\
\hline Trimethoprim-sulfamethoxazole & $\begin{array}{c}\text { Trimethoprim- } \\
\text { Sulfamethoxazole }(S X T)^{a}\end{array}$ & 25 & $\leq 10$ & 0 & $35(29.21)$ \\
\hline Lincosamide & Clindamycin $(C L I)^{a}$ & 2 & $\leq 14$ & $3(15.79)$ & $50(42.73)$ \\
\hline Cephalosporins & Cefoxitin (FOX) ${ }^{a}$ & 30 & $\leq 24$ & $4(21.05)$ & $9(7.69)$ \\
\hline Tetracyclines & Doxycycline $(D O X)^{a}$ & 30 & $\leq 12$ & $\begin{array}{c}2(100) \\
0\end{array}$ & 0 \\
\hline \multirow[t]{2}{*}{ Penicillins } & Penicillin $(P E N)^{a}$ & 6 & $\leq 28$ & 17(89.47) & $104(88.89)$ \\
\hline & Oxacillin ${ }^{b}$ & - & $\geq 0.5 / \geq 4^{c}$ & $4(21.05)$ & $19(16.23)$ \\
\hline \multirow[t]{3}{*}{ Aminoglycosides } & Kanamycin $(K A N)^{a}$ & 30 & $\leq 13$ & $3(15.79)$ & $58(49.57)$ \\
\hline & Amikacin $(A M K)^{a}$ & 30 & $\leq 14$ & $3(15.79)$ & $1(0.85)$ \\
\hline & Gentamicin (GEN) ${ }^{a}$ & 10 & $\leq 12$ & $3(15.79)$ & $28(23.93)$ \\
\hline \multirow[t]{2}{*}{ Macrolides } & Erythromycin $(E R Y)^{a}$ & 15 & $\leq 13$ & $5(26.31)$ & $54(46.15)$ \\
\hline & Clarithromycin (CLR) ${ }^{a}$ & 15 & $\leq 13$ & $5(26.31)$ & $54(46.15)$ \\
\hline Linezolid & Linezolid (LZD) $a$ & 30 & $\leq 20$ & 0 & 0 \\
\hline Rifampin & Rifampin $(R I F)^{a}$ & RIF5 & $\leq 16$ & 0 & 0 \\
\hline Vancomycin. & Vancomycin $b$ & - & $\geq 32 / \geq 16^{\mathrm{d}}$ & 0 & 0 \\
\hline
\end{tabular}

a Antibiotic susceptibility was determined by the disk diffusion technique; zone diameters were expressed in $\mathrm{mm} .{ }^{\mathrm{b}} \mathrm{MIC}$ sere determined by agar dilution and are expressed in $\mu \mathrm{g} / \mathrm{mL}$.

Breakpoint for Coagulase-negative Staphylococcus (0.5) and S. aureus (4). ${ }^{\mathrm{d}}$ Breakpoint for Coagulase-negative Staphylococcus (32) and S. aureus (16). 
Table 3. Rates of antimicrobial resistance of 3 S. schleiferi, 19 S. aureus and 117 S. pseudintermedius isolates recovered at the Complutense Veterinary Teaching Hospital (Spain) during different periods (2007-2010, $n=44 ; 2011-2014, n=44$ and 2015-2016, $n=51)$.

\begin{tabular}{|c|c|c|c|c|}
\hline \multirow{2}{*}{$\begin{array}{c}\text { Antibiotic } \\
\text { (Abbreviation) }\end{array}$} & \multirow{2}{*}{$\begin{array}{c}\text { Disk Content } \\
(\mu \mathrm{g})\end{array}$} & \multicolumn{3}{|c|}{ Number of Resistant (\% Resistance) } \\
\hline & & $\begin{array}{c}2007-2010 \\
(\mathrm{n}=44)\end{array}$ & $\begin{array}{c}2011-2014 \\
(n=43)\end{array}$ & $\begin{array}{c}2015-2016 \\
(n=51)\end{array}$ \\
\hline Kanamycin (KAN) & 30 & $15(34.09 \%)$ & $20(45.45 \%)$ & $26(50.98 \%)$ \\
\hline Amikacin $(A M K)$ & 30 & $1(2.27 \%)$ & $2(4.55 \%)$ & $1(1.96 \%)$ \\
\hline Gentamicin (GEN) & 10 & $6(13.64 \%)$ & $10(22.73 \%)$ & $15(29.41 \%)$ \\
\hline Cefoxitin (FOX) & 30 & $3(6.82 \%)$ & $6(13.64 \%)$ & $4(7.84 \%)$ \\
\hline Penicillin (PEN) & 6 & $38(86.36 \%)$ & $36(81.82 \%)$ & $47(92.16 \%)$ \\
\hline Ciprofloxacin (CIP) & 5 & $4(9.09 \%)$ & $8(18.18 \%)$ & $15(29.41 \%)$ \\
\hline Norfloxacin (NOR) & 10 & $5(11.36 \%)$ & $8(18.18 \%)$ & $15(29.41 \%)$ \\
\hline Ofloxacin (OFX) & 5 & $4(9.09 \%)$ & $8(18.18 \%)$ & $15(29.41 \%)$ \\
\hline Tetracycline (TET) & 30 & $21(47.73 \%)$ & $22(50 \%)$ & $19(37.25 \%)$ \\
\hline Erythromycin (ERY) & 15 & $18(40.91 \%)$ & $17(38.64 \%)$ & $24(47.06 \%)$ \\
\hline Clarithromycin (CLR) & 15 & $18(40.91 \%)$ & $17(38.64 \%)$ & $24(47.06 \%)$ \\
\hline Teicoplanin (TEC) & 30 & 0 & 0 & 0 \\
\hline Doxycycline (DOX) & 30 & 0 & 0 & 0 \\
\hline $\begin{array}{c}\text { Trimethoprim- } \\
\text { Sulfamethoxazole (SXT) }\end{array}$ & 25 & $4(9.09 \%)$ & $16(36.36 \%)$ & $15(29.41 \%)$ \\
\hline Linezolid (LZD) & 30 & 0 & 0 & 0 \\
\hline Rifampin (RIF) & 5 & $15(34.09 \%)$ & $17(38.64 \%)$ & $21(41.18 \%)$ \\
\hline Clindamycin (CLI) & 2 & 0 & 0 & 0 \\
\hline Chloramphenicol (CHL) & 30 & $10(22.73 \%)$ & $8(18.18 \%)$ & $11(21.57 \%)$ \\
\hline
\end{tabular}

\subsection{Genomic Characterization of the Methicillin-Resistant Coagulase-Positive Staphylococcus (MRCoPS)}

A total of 20 CoPS isolates resistant to oxacillin and with a positive result in the ALERE PBP2a test (17 S. pseudintermedius and 3 S. aureus isolates) were subjected to molecular analysis. All the strains were isolated from dogs, except $\mathrm{S}$. aureus strain $94 \mathrm{~S}$, which was isolated from a pig's lung. There was a significant association between MDR and methicillin resistance for S. pseudintermedius isolates $(P<0.05$, Fisher's exact test). MRSP and MSSP isolates showed resistance against the same antibiotics except in the case of amikacin, which was only detected in one MRSP isolate. Eighteen isolates (90\%) were found to possess the methicillin resistance-encoding mecA gene (this gene was not found in the genomes of S. pseudointermedius isolates $125 \mathrm{~S}$ and 134S) (Supplementary Material). We performed an in silico Staphylococcal cassette chromosome mec (SCCmec) analysis of the 18 mecA-containing isolates and found that most S. pseudointermedius isolates belonged to the SCCmec III type (3A) (13 isolates, 86.7\%), whereas two isolates belonged to the SCCmec V type (13.3\%). Likewise, three different sequence types (STs) were detected among MRSP isolates: ST71 (15 isolates, 88.23\%), ST196 (1 isolate, 5.88\%) and ST261 (1 isolate, 5.88\%). Interestingly, there was a perfect correlation between SCCmec types and STs, as all SCCmec III (3A) isolates belonged to ST71, whereas MRSP isolates of the SCCmec V (2B) and SCCmec V types belonged to STs 261 and 196, respectively. Regarding the MLST results of MRSA isolates, one of the isolates (94S) belonged to ST398, which is the ST typically associated with the livestock-associated MRSA (LA-MRSA) lineage, whereas the other two MRSA isolates belonged to two other different lineages, namely, community-associated MRSA (CA-MRSA) lineage (isolate 55S, ST8), and hospital-associated MRSA (HA-MRSA) lineage (16S, ST125).

The molecular AMR profiles of 20 MRCoPS are summarized in Table S1 (see Supplementary Material). A total of 17 out of $20(85 \%)$ MRCoPS isolates carried the $\beta$-lactamase gene blaZ. The presence of genes encoding resistance to macrolides (clarithromycin and erythromycin) were detected in 18 MRCoPS isolates (90\%) including $15(75 \%)$ methylase gene erm(B), and $2(10 \%)$ methylase gene erm $(C)$. In addition, the $A B C$ transporter-encoding gene $m s r A$ and modifying enzyme $m p h C$ gene were found in the same MRSA isolate, while $\operatorname{erm}(B)$ or $\operatorname{erm}(C)$ genes were not detected. Macrolide 
resistance-encoding genes were not detected in two MRSP. Moreover, detection of lincosamide nucleotidyl transferase-encoding gene, $\ln u(A)$ and $\operatorname{lin} A$ genes were detected in only one MRSA isolate. Membrane-associated efflux pump genes tet $(K)$ and ribosome binding site gene tet $(M)$ were detected in five $(25 \%)$ and three (15\%) MRCoPS isolates. One MRSA isolate simultaneously carried tet $(K)$ and tet $(M)$ genes.

Results for aminoglycoside-streptothricin resistance-encoding genes are summarized in Table S1. Almost all MRCoPS isolates $(17,85 \%)$ carried the kanamycin resistance gene aph(3'). In at least $90 \%$ of isolates, two aminoglycoside-streptothricin resistance-encoding genes were simultaneously detected with ant $(6)+a p h\left(3^{\prime}\right)+\operatorname{aad}(6)+S A T-4$ being the most frequent combination (15 out of 20) among MRCoPS in our study. The cat(pc221) chloramphenicol acetyltransferase gene was detected in the four chloramphenicol-resistant MRSP isolates. Finally, $80 \%$ of the isolates (16 MRSP) carried the trimethoprim resistance gene $d f r(G)$.

\section{Discussion}

Staphylococcus pseudintermedius has been identified as the most important SIG pathogen in dogs [1], although it may also be isolated from other animal species [13] and humans [14]. Furthermore, MRSP is frequently recovered from pets with pyoderma and healthy animals [9]. In this context, reliable and accurate species-level identification of $S$. pseudintermedius is key to the implementation of control measures for successful treatment and disease management [15]. Additionally, correct identification of S. pseudintermedius may help to clarify the current clinical role of this species in veterinary medicine [16].

In this study, S. pseudintermedius was the only SIG species found among canine isolates, which agrees with the results of previous studies [17]. Different authors have demonstrated that S. pseudintermedius is frequently misidentified as S. intermedius by phenotypic methods [5]. Indeed, 13 S. pseudintermedius isolates from our strain collection were misidentified as $S$. aureus and 85 as $S$. intermedius by the VITEK system. A possible explanation for these discrepancies could be the lack of $\beta$-galactosidase test in the VITEK panel, which is frequently used to differentiate between $S$. pseudintermedius and S. aureus [18]. Similarly, S. pseudintermedius isolates can be misidentified as $S$. intermedius based on mannitol fermentation tests [19]. Previous reports have recognized the nuc-based multiplex PCR assay described by Sasaki et al [5] as the gold standard method to differentiate CoPS species [20]. The suitability of MALDI-TOF approaches for species-level differentiation of SIG members has also been suggested [21], although certain limitations in identifying S. pseudintermedius were reported [20]. Accordingly, four out of 117 S. pseudintermedius isolates included in this study were misidentified as S. intermedius by PCR.

Regarding the genetic diversity of the MRSP isolates characterized in this study, it is notable that most of them (15 out of 17 isolates, i.e., 88.2\%) belonged to the same sequence type (ST71), which is perfectly correlated with the SECCmec III(3A) type in those isolates containing the mecA gene. This contrasts with the results of previous studies that found a higher genetic diversity for $S$. pseudintermedius (e.g., 24 different STs out of 69 MRSP isolates and 11 STs out of 23 MRSP isolates [9,22]. The high prevalence of the ST71 genotype among the S. pseudintermedius isolates recovered from dogs in Spain has been previously reported [10]. Moreover, in some cases, it has been demonstrated that MRSP are often genetically more homogeneous than MSSP [23]. The ST71 clonal group has been formerly considered as epidemic in Europe, but this multidrug resistant lineage has also spread to other regions [24]. With respect to the two other MRSP genotypes detected in this study, ST196 has been found in Portugal [8]. On the other hand, molecular characterization revealed that our MRSA isolates belonged to three different STs (one isolate each), namely, ST398, ST128 and ST8, which represent two different origins: animal (ST398 belongs to the LA-MRSA linage), and human (ST8 and ST125 belong to CA-MRSA and HA-MRSA lineage, respectively). These findings on MRSA suggest that while isolate 94S, isolated from a pig's lung, is a natural animal MRSA strain, the other two MRSA isolates isolated from dogs might have been transferred from the animals' owners. 
S. pseudintermedius isolates had the greatest levels of resistance to penicillin (104/117 isolates, i.e., $88.8 \%$ ). The resistance rates to fluoroquinolones detected in the present study (around 20\%) are in accordance with previous reports [25].

The methicillin resistance of our selected isolates was confirmed by a PBP2a-based culture colony test, which has been recognized as a suitable diagnostic test to detect beta-lactam-resistant SIG isolates [26]. Methicillin-resistant S. aureus and S. pseudintermedius have emerged in the last decade. The relevance of MRSP in veterinary clinics is related to their frequent multidrug resistance, even to all licensed drugs available for dogs [1]. In addition, MRSP is considered a nosocomial pathogen in veterinary care settings [27]. In particular, around $16 \%$ of the CoPS isolates recovered from veterinary patients and characterized in the present study were methicillin-resistant, thus confirming MRSP circulation among Spanish veterinary patients. The prevalence of MRSP found in our study is higher than the figures previously reported in Spain (1\% in healthy household-dogs and $8 \%$ in healthy pound-dogs [10]). In contrast, high rates of MRSP isolation from veterinary patients have been reported in other countries [28].

Different studies have highlighted the emergence of multidrug-resistant CoPS [29], which were detected in $47.48 \%$ of isolates in the present study. Of these, 19 isolates were MRSP (out of 19, that is, $100 \%)$. MRSP are commonly associated with MDR profiles. For example, Gomez-Sanz et al. [10] found that all nine MRSP identified among 196 CoPS tested isolates were MDR. Different studies have also detected MDR among MSSP isolates [8]. MSSP isolates carried similar antibiotic resistance profiles to those previously reported in Spain showing high rates of resistances against $\beta$-lactams, macrolides, lincosamide and aminoglycosides $[10,11]$. In our study, $41.84 \%$ of MSSP isolates showed a multidrug-resistant profile, which is similar to $39 \%$ of MSSP isolates previously reported in Spain [11]. MSSP isolates are usually resistant to penicillin/ampicillin and tetracycline [30,31]. However, in our study, MSSP showed resistance to the same antimicrobials as MRSP except amikacin. This latter result agrees with that reported by Gold et al. [32], who found that resistance to amikacin was linked to methicillin-resistance.

Most antibiotic resistance genes detected in the genome of our collection of MRSP isolates have been reported by other authors and are presumed to be acquired via transposon transfer from other Gram-positive bacteria [33]. However, the genetic basis of some of the resistant phenotypes found in this study could not be determined. Previous studies have revealed discrepancies between antibiotic resistant phenotypes and those inferred from genetic information, particularly among isolates from some clonal complexes such as CC71 [34]. The lack of resistance breakpoints for veterinary isolates of S. pseudintermedius may contribute to these discrepancies [34].

The presence of the mecA gene in the genome of $S$. pseudintermedius isolates has been previously reported [29]. In our study, all but two (15/17, i.e., 88.2\%) methicillin-resistant CoPS isolates had such antibiotic resistance markers. With regard to genes linked to tetracycline resistance, tet $(K)$ gene was the most frequent gene in the set of MRSP isolates included in this study, which agrees with the results obtained by other authors (e.g., Perreten et al. [1]). It has been suggested that MRSP ST71 only showed tet $(K)$ [8], which would explain its presence in most of our isolates. Actually, tet $(M)$ was only present in the ST196 and ST261 isolates (one isolate each) that we found, and tet $(L)$, previously found in MSSP isolates from Spanish dogs [12], was not detected in any of the isolates characterized in this study.

As in other studies, the cat(PC221)-carrying plasmid was the most frequent mechanism of chloramphenicol resistance identified in our collection of $S$. pseudintermedius isolates [1]. As previously observed in Europe and North America, most of our MRSP and MRSA isolates carried the blaZ gene. Furthermore, the $\operatorname{aph}\left(3^{\prime}\right)$ gene, which encodes an amynoglycoside-modyfying enzyme was the most frequent aminoglycoside resistance encoding gene found in isolates from dogs, thus agreeing with previous reports [32]. Remarkably, the streptomycin adenyltransferase encoding gene ant(6), the kanamycin resistance-encoding gene $a p h\left(3^{\prime}\right)$, and the streptothricin acetyltransferase encoding gene sat -4 were simultaneously detected in $65 \%$ of our CoPS isolates, which is a lower frequency than in previous reports from Spain [10] and other countries [1]. Previous studies have also detected the rRNA 
methylase-encoding gene $\operatorname{erm}(B)$ as the most frequent marker of macrolide/lincosamide resistance [35]. The dihydrofolate reductase-encoding gene $d f r(G)$ was detected in most trimethoprim-resistant MSRA and MRSP isolates as previously found [10,13].

There is a notably high degree of heterogeneity in antibiotic resistance results across different studies focused on S. pseudintermedius, which may be due to the lack of harmonized strategies for antimicrobial susceptibility testing and the interpretation of resistance, as previously suggested by Moodley et al. [30]. Our study highlights the emergence of antibiotic resistance, particularly to fluoroquinolones and trimethoprim-sulfamethoxazole, for which an increasing resistance over time was demonstrated in CoPS isolates recovered from animals in Spain. The establishment of harmonized tools for CoPS surveillance may help to monitor the emergence of AMR in different countries, which is a key step for implementing adequate antibiotic management practices in veterinary clinics.

\section{Materials and Methods}

\subsection{Samples and Isolates}

A total of 5468 clinical samples were subjected to bacteriological analysis at the Veterinary Teaching Hospital of Complutense University of Madrid (Madrid, Spain) from 2007 to 2016. Staphylococcus spp. was identified in 842 samples (15.4\%) according to VITEK results (BioMèrieux, Marcy-1'Etoile, France). Of these, 139 isolates (16.5\% of the total number of Staphylococcus isolates) were randomly selected for the present study for species-level identification by different bioanalytical techniques and subsequent antimicrobial susceptibility testing (as detailed below).

\subsection{Bacterial Identification}

After VITEK identification, all studied isolates were subjected to MALDI-TOF mass spectrometry analysis, following the procedures detailed in Perez-Sancho et al. [36]. Briefly, isolates were grown on Columbia blood agar plates (bioMérieux) at $37^{\circ} \mathrm{C}$ for $24 \mathrm{~h}$. Proteins were extracted following the manufacturer's instructions (Bruker Daltonik, Bremen, Germany). Spectra acquisition was performed automatically using an UltrafleXtreme device (Bruker Daltonik) in the linear and positive mode. Species-level identification was achieved by comparing the MALDI profile of isolates with those included in the BDAL Bruker database (5989 entries, accessed on April 2016) using MALDI Biotyper software (Bruker Daltonik).

Additionally, SIG isolates were also identified by a multiplex PCR based on thermonuclease-encoding $(n u c)$ gene [5], which is considered to be the gold standard technique for SIG species-level identification [20,37]. On the contrary, S. aureus isolates were only identified by MALDI-TOF MS, as this technique achieves reliable identification of this Staphylococcus species [38,39].

\subsection{Antimicrobial Susceptibility Testing}

Antibiotic susceptibility was assessed by the disk diffusion method following the Clinical and Laboratory Standard Institute (CLSI) guidelines [40-42]. MICs to vancomycin and oxacillin were determined by the agar dilution method, which was performed according to CLSI's instructions [41]. Oxacillin-resistant isolates were subjected to the ALERE PBP2a culture colony test (Alere Healthcare, L'Hospitalet de Llobregat, Barcelona), which is an immunochromatographic membrane assay that allows the detection of the penicillin-binding protein PBP2a in clinical isolates in approximately 5 min. Staphylococcus aureus ATCC 25923 was included as the quality control strain in all these analyses.

\subsection{Molecular Analysis of Methicillin Resistant CoPS}

All isolates resistant to oxacillin and with a positive result in the ALERE PBP2a test were subjected to whole genome sequencing (WGS) for further characterization of their antibiotic resistance determinants. Bacterial strains were sequenced on a MiSeq (Illumina) platform as previously described [43]. Briefly, genomic DNA was purified from pure cultures with the QIAGEN DNeasy Blood and Tissue Kit, 
and sequencing libraries were prepared using the Nextera XT kit and sequenced using v3 reagents with

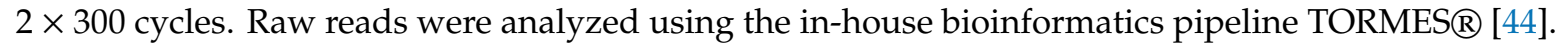
Briefly, it consists of quality filtering by Prinseq v.0.20.4 [45] and Trimmomatic [46]. Genomes were assembled by using SPAdes v3.10 [47], and classified taxonomically and annotated by prokka [48]. The profiles of multi-locus sequencing typing (MLST) were predicted by using mlst v2.10 (T. Seemann, https://github.com/tseemann/mlst) against the PubMLST database [49]. Searching SCCmec was performed using SCCmecFinder (https://cge.cbs.dtu.dk/services/SCCmecFinder/). The resistomes inferred from the draft genomes were analyzed by BLASTN [50] and ABRicate (T. Seemann, https: //github.com/tseemann/abricate) searches against the ResFinder [51] and CARD [52]. The presence of virulence genes was inferred by BLASTN searches against the Virulence Factors DataBase (VFDB) [53].

\subsection{Data Analysis}

The association between Staphylococcus species (S. aureus and S. pseudintermedius) and AMR frequencies was assessed using Fisher's exact test. The relationship between the origin of the sample (body fluids, ear exudates and other origins) and AMR were also evaluated using Fisher's exact and multiple proportions Z-test with Bonferroni adjustment for multiple comparisons, respectively. The association between the resistance rates to the different antibiotics tested was determined by Cohen's kappa coefficient $(\kappa)$. The Cochran-Armitage trend test was used to determine the increase in different AMR over time. Statistical tests were performed using SPSS 25 software (IBM, New York, NY, USA), except the Cochran-Armitage trend test, which was performed with the WinPepi package [54]. Statistical significance was established at $P<0.05$.

\section{Conclusions}

In conclusion, the results of this study confirm the value of MALDI-TOF technologies for species-level identification of SIG isolates of animal origin. Furthermore, we observed a high prevalence of MDR and resistance to fluoroquinolones, cephalosporins and macrolides, and detected epidemic MRSP genotypes such as ST71 among isolates from dogs. Finally, we found a variety of genes that have been related to antibiotic-resistant phenotypes in the genome of MRSP isolates. All in all, these results highlight the emerging threat posed by MRSP and the need for continuous surveillance of this bacterial group for antimicrobial resistance in the veterinary setting.

Supplementary Materials: The following are available online at http://www.mdpi.com/2079-6382/9/11/752/s1, Table S1: Phenotypes and molecular AMR profiles of 20 Methicillin-Resistant Coagulase Positive Staphylococcus recovered from animals in Spain.

Author Contributions: Conceptualization, M.P.-S., J.L.B., M.E.G., S.A.-P.; methodology, S.A.-P., M.P.-S., T.G.-S., M.H.; software, M.H., D.R.-L. Writing—original draft preparation, M.P.-S., T.G.-S., S.A.-P., J.L.B.; writing一review and editing, J.L.B., M.E.G., M.H., D.R.-L., L.D.; supervision, J.L.B., M.E.G., L.D. All authors have read and agreed to the published version of the manuscript.

Funding: Sergio Alvarez-Perez acknowledges a 'Ramón y Cajal' contract funded by the Spanish Ministry of Science and Innovation [RYC2018-023847-I]. The funders had no role in the preparation of the manuscript or decision to publish.

Conflicts of Interest: The authors declare no conflict of interest.

\section{References}

1. Perreten, V.; Kadlec, K.; Schwarz, S.; Gronlund Andersson, U.; Finn, M.; Greko, C.; Moodley, A.; Kania, S.A.; Frank, L.A.; Bemis, D.A.; et al. Clonal spread of methicillin-resistant Staphylococcus pseudintermedius in Europe and North America: An international multicentre study. J. Antimicrob. Chemother. 2010, 65, 1145-1154. [CrossRef] [PubMed]

2. Ruscher, C.; Lubke-Becker, A.; Wleklinski, C.G.; Soba, A.; Wieler, L.H.; Walther, B. Prevalence of Methicillin-resistant Staphylococcus pseudintermedius isolated from clinical samples of companion animals and equidaes. Vet. Microbiol. 2009, 136, 197-201. [CrossRef] 
3. Viau, R.; Hujer, A.M.; Hujer, K.M.; Bonomo, R.A.; Jump, R.L. Are Staphylococcus intermedius Infections in Humans Cases of Mistaken Identity? A Case Series and Literature Review. Open Forum Infect. Dis. 2015, 2, ofv110. [CrossRef]

4. Pires Dos Santos, T.; Damborg, P.; Moodley, A.; Guardabassi, L. Systematic Review on Global Epidemiology of Methicillin-Resistant Staphylococcus pseudintermedius: Inference of Population Structure from Multilocus Sequence Typing Data. Front. Microbiol. 2016, 7, 1599. [CrossRef] [PubMed]

5. Sasaki, T.; Tsubakishita, S.; Tanaka, Y.; Sakusabe, A.; Ohtsuka, M.; Hirotaki, S.; Kawakami, T.; Fukata, T.; Hiramatsu, K. Multiplex-PCR method for species identification of coagulase-positive staphylococci. J. Clin. Microbiol. 2010, 48, 765-769. [CrossRef] [PubMed]

6. Blaiotta, G.; Fusco, V.; Ercolini, D.; Pepe, O.; Coppola, S. Diversity of Staphylococcus species strains based on partial kat (catalase) gene sequences and design of a PCR-restriction fragment length polymorphism assay for identification and differentiation of coagulase-positive species (S. aureus, S. delphini, S. hyicus, S. intermedius, S. pseudintermedius, and S. schleiferi subsp. coagulans). J. Clin. Microbiol. 2010, 48, 192-201. [PubMed]

7. Kmieciak, W.; Szewczyk, E.M.; Ciszewski, M. Searching for Beta-Haemolysin hlb Gene in Staphylococcus pseudintermedius with Species-Specific Primers. Curr. Microbiol. 2016, 73, 148-152. [CrossRef]

8. Couto, N.; Belas, A.; Couto, I.; Perreten, V.; Pomba, C. Genetic relatedness, antimicrobial and biocide susceptibility comparative analysis of methicillin-resistant and -susceptible Staphylococcus pseudintermedius from Portugal. Microb. Drug Resist. 2014, 20, 364-371. [CrossRef] [PubMed]

9. Feng, Y.; Tian, W.; Lin, D.; Luo, Q.; Zhou, Y.; Yang, T.; Deng, Y.; Liu, Y.H.; Liu, J.H. Prevalence and characterization of methicillin-resistant Staphylococcus pseudintermedius in pets from South China. Vet. Microbiol. 2012, 160, 517-524. [CrossRef] [PubMed]

10. Gomez-Sanz, E.; Torres, C.; Lozano, C.; Saenz, Y.; Zarazaga, M. Detection and characterization of methicillin-resistant Staphylococcus pseudintermedius in healthy dogs in La Rioja, Spain. Comp. Immunol. Microbiol. Infect. Dis. 2011, 34, 447-453. [CrossRef]

11. Gomez-Sanz, E.; Torres, C.; Lozano, C.; Zarazaga, M. High diversity of Staphylococcus aureus and Staphylococcus pseudintermedius lineages and toxigenic traits in healthy pet-owning household members. Underestimating normal household contact? Comp. Immunol. Microbiol. Infect. Dis. 2013, 36, 83-94. [CrossRef]

12. Gomez-Sanz, E.; Torres, C.; Benito, D.; Lozano, C.; Zarazaga, M. Animal and human Staphylococcus aureus associated clonal lineages and high rate of Staphylococcus pseudintermedius novel lineages in Spanish kennel dogs: Predominance of S. aureus ST398. Vet. Microbiol. 2013, 166, 580-589. [CrossRef] [PubMed]

13. Kadlec, K.; Schwarz, S.; Perreten, V.; Andersson, U.G.; Finn, M.; Greko, C.; Moodley, A.; Kania, S.A.; Frank, L.A.; Bemis, D.A.; et al. Molecular analysis of methicillin-resistant Staphylococcus pseudintermedius of feline origin from different European countries and North America. J. Antimicrob. Chemother. 2010, 65, 1826-1828. [CrossRef]

14. Van Hoovels, L.; Vankeerberghen, A.; Boel, A.; Van Vaerenbergh, K.; De Beenhouwer, H. First case of Staphylococcus pseudintermedius infection in a human. J. Clin. Microbiol. 2006, 44, 4609-4612. [CrossRef] [PubMed]

15. Limbago, B.M. What's in a Name? The Impact of Accurate Staphylococcus pseudintermedius Identification on Appropriate Antimicrobial Susceptibility Testing. J. Clin. Microbiol. 2016, 54, 516-517. [CrossRef] [PubMed]

16. Weese, J.S.; van Duijkeren, E. Methicillin-resistant Staphylococcus aureus and Staphylococcus pseudintermedius in veterinary medicine. Vet. Microbiol. 2010, 140, 418-429. [CrossRef]

17. Devriese, L.A.; Hermans, K.; Baele, M.; Haesebrouck, F. Staphylococcus pseudintermedius versus Staphylococcus intermedius. Vet. Microbiol. 2009, 133, 206-207. [CrossRef]

18. Roberson, J.R.; Fox, L.K.; Hancock, D.D.; Besser, T.E. Evaluation of methods for differentiation of coagulase-positive staphylococci. J. Clin. Microbiol. 1992, 30, 3217-3219. [CrossRef]

19. Riegel, P.; Jesel-Morel, L.; Laventie, B.; Boisset, S.; Vandenesch, F.; Prevost, G. Coagulase-positive Staphylococcus pseudintermedius from animals causing human endocarditis. Int. J. Med. Microbiol. 2011, 301, 237-239. [CrossRef]

20. Silva, M.B.; Ferreira, F.A.; Garcia, L.N.; Silva-Carvalho, M.C.; Botelho, L.A.; Figueiredo, A.M.; Vieira-da-Motta, O. An evaluation of matrix-assisted laser desorption ionization time-of-flight mass spectrometry for the identification of Staphylococcus pseudintermedius isolates from canine infections. J. Vet. Diagn. Invest. 2015, 27, 231-235. [CrossRef] 
21. Decristophoris, P.; Fasola, A.; Benagli, C.; Tonolla, M.; Petrini, O. Identification of Staphylococcus intermedius Group by MALDI-TOF MS. Syst. Appl. Microbiol. 2011, 34, 45-51. [CrossRef] [PubMed]

22. Osland, A.M.; Vestby, L.K.; Fanuelsen, H.; Slettemeas, J.S.; Sunde, M. Clonal diversity and biofilm-forming ability of methicillin-resistant Staphylococcus pseudintermedius. J. Antimicrob. Chemother. 2012, 67, 841-848. [CrossRef] [PubMed]

23. Black, C.C.; Solyman, S.M.; Eberlein, L.C.; Bemis, D.A.; Woron, A.M.; Kania, S.A. Identification of a predominant multilocus sequence type, pulsed-field gel electrophoresis cluster, and novel staphylococcal chromosomal cassette in clinical isolates of mecA-containing, methicillin-resistant Staphylococcus pseudintermedius. Vet. Microbiol. 2009, 139, 333-338. [CrossRef] [PubMed]

24. Wang, Y.; Yang, J.; Logue, C.M.; Liu, K.; Cao, X.; Zhang, W.; Shen, J.; Wu, C. Methicillin-resistant Staphylococcus pseudintermedius isolated from canine pyoderma in North China. J. Appl. Microbiol. 2012, 112, 623-630. [CrossRef] [PubMed]

25. Yoo, J.H.; Yoon, J.W.; Lee, S.Y.; Park, H.M. High prevalence of Fluoroquinolone- and Methicillin-resistant Staphylococcus pseudintermedius isolates from canine pyoderma and otitis externa in veterinary teaching hospital. J. Microbiol. Biotechnol. 2010, 20, 798-802.

26. Arnold, A.R.; Burnham, C.A.; Ford, B.A.; Lawhon, S.D.; McAllister, S.K.; Lonsway, D.; Albrecht, V.; Jerris, R.C.; Rasheed, J.K.; Limbago, B.; et al. Evaluation of an Immunochromatographic Assay for Rapid Detection of Penicillin-Binding Protein 2a in Human and Animal Staphylococcus intermedius Group, Staphylococcus lugdunensis, and Staphylococcus schleiferi Clinical Isolates. J. Clin. Microbiol. 2016, 54, 745-748. [CrossRef]

27. Sasaki, T.; Kikuchi, K.; Tanaka, Y.; Takahashi, N.; Kamata, S.; Hiramatsu, K. Methicillin-resistant Staphylococcus pseudintermedius in a veterinary teaching hospital. J. Clin. Microbiol. 2007, 45, 1118-1125. [CrossRef]

28. Kawakami, T.; Shibata, S.; Murayama, N.; Nagata, M.; Nishifuji, K.; Iwasaki, T.; Fukata, T. Antimicrobial susceptibility and methicillin resistance in Staphylococcus pseudintermedius and Staphylococcus schleiferi subsp. coagulans isolated from dogs with pyoderma in Japan. J. Vet. Med. Sci. 2010, 72, 1615-1619. [CrossRef]

29. Van Duijkeren, E.; Catry, B.; Greko, C.; Moreno, M.A.; Pomba, M.C.; Pyorala, S.; Ruzauskas, M.; Sanders, P.; Threlfall, E.J.; Torren-Edo, J.; et al. Review on methicillin-resistant Staphylococcus pseudintermedius. J. Antimicrob. Chemother. 2011, 66, 2705-2714. [CrossRef]

30. Moodley, A.; Damborg, P.; Nielsen, S.S. Antimicrobial resistance in methicillin susceptible and methicillin resistant Staphylococcus pseudintermedius of canine origin: Literature review from 1980 to 2013. Vet. Microbiol. 2014, 171, 337-341. [CrossRef]

31. Siak, M.; Burrows, A.K.; Coombs, G.W.; Khazandi, M.; Abraham, S.; Norris, J.M.; Weese, J.S.; Trott, D.J. Characterization of meticillin-resistant and meticillin-susceptible isolates of Staphylococcus pseudintermedius from cases of canine pyoderma in Australia. J. Med. Microbiol. 2014, 63, 1228-1233. [CrossRef]

32. Gold, R.M.; Cohen, N.D.; Lawhon, S.D. Amikacin resistance in Staphylococcus pseudintermedius isolated from dogs. J. Clin. Microbiol. 2014, 52, 3641-3646. [CrossRef] [PubMed]

33. Kadlec, K.; Schwarz, S. Antimicrobial resistance of Staphylococcus pseudintermedius. Vet. Dermatol. 2012, 23, 276-282 e55. [CrossRef]

34. Wegener, A.; Broens, E.M.; Zomer, A.; Spaninks, M.; Wagenaar, J.A.; Duim, B. Comparative genomics of phenotypic antimicrobial resistances in methicillin-resistant Staphylococcus pseudintermedius of canine origin. Vet. Microbiol. 2018, 225, 125-131. [CrossRef] [PubMed]

35. Boerlin, P.; Burnens, A.P.; Frey, J.; Kuhnert, P.; Nicolet, J. Molecular epidemiology and genetic linkage of macrolide and aminoglycoside resistance in Staphylococcus intermedius of canine origin. Vet. Microbiol. 2001, 79, 155-169. [CrossRef]

36. Perez-Sancho, M.; Vela, A.I.; Kostrzewa, M.; Zamora, L.; Casamayor, A.; Dominguez, L.; Fernandez-Garayzabal, J.F. First analysis by MALDI-TOF MS technique of Chryseobacterium species relevant to aquaculture. J. Fish Dis. 2018, 41, 389-393. [CrossRef] [PubMed]

37. Wu, M.T.; Burnham, C.A.; Westblade, L.F.; Dien Bard, J.; Lawhon, S.D.; Wallace, M.A.; Stanley, T.; Burd, E.; Hindler, J.; Humphries, R.M. Evaluation of Oxacillin and Cefoxitin Disk and MIC Breakpoints for Prediction of Methicillin Resistance in Human and Veterinary Isolates of Staphylococcus intermedius Group. J. Clin. Microbiol. 2016, 54, 535-542. [CrossRef]

38. Elbehiry, A.; Al-Dubaib, M.; Marzouk, E.; Osman, S.; Edrees, H. Performance of MALDI biotyper compared with Vitek 2 compact system for fast identification and discrimination of Staphylococcus species isolated from bovine mastitis. MicrobiologyOpen 2016, 5, 1061-1070. [CrossRef] 
39. Saraiva, M.M.; De Leon, C.M.; Santos, S.C.; Stipp, D.T.; Souza, M.M.; Santos Filho, L.; Gebreyes, W.A.; Oliveira, C.J. Accuracy of PCR targeting different markers for Staphylococcus aureus identification: A comparative study using matrix-assisted laser desorption/ionization time-of-flight mass spectrometry as the gold standard. J. Vet. Diagn. Investig. 2018, 30, 252-255. [CrossRef]

40. Clinical and Laboratory Standards Institute. Performance Standards for Antimicrobial Disk Susceptibility Tests, 13th ed.; CLSI Standard M02; Clinical and Laboratory Standards Institute: Wayne, PA, USA, 2018.

41. Clinical and Laboratory Standards Institute. Methods for Dilution Antimicrobial Susceptibility Tests for Bacteria That Grow Aerobically, 11th ed.; CLSI Standard M07; Clinical and Laboratory Standards Institute: Wayne, PA, USA, 2018.

42. Clinical and Laboratory Standards Institute. Performance Standards for Antimicrobial Susceptibility Testing, 30th ed.; CLSI Standard M100; Clinical and Laboratory Standards Institute: Wayne, PA, USA, 2020.

43. Hernández, M.; Iglesias, M.R.; Rodríguez-Lázaro, D.; Gallardo, A.; Quijada, N.; Miguela-Villoldo, P.; Campos, M.J.; Píriz, S.; López-Orozco, G.; de Frutos, C.; et al. Co-occurrence of colistin-resistance genes $m c r-1$ and $m c r-3$ among multidrug-resistant Escherichia coli isolated from cattle, Spain, September 2015. Eurosurveillance 2017, 22, 30586. [CrossRef]

44. Quijada, N.M.; Rodríguez-Lázaro, D.; Eiros, J.M.; Hernandez, M. TORMES: An automated pipeline for whole bacterial genome analysis. Bioinformatics 2019, 35, 4207-4212. [CrossRef] [PubMed]

45. Schmieder, R.; Edwards, R. Quality control and preprocessing of metagenomic datasets. Bioinformatics 2011, 27, 863-864. [CrossRef] [PubMed]

46. Bolger, A.M.; Lohse, M.; Usadel, B. Trimmomatic: A flexible trimmer for Illumina sequence data. Bioinformatics 2014, 30, 2114-2120. [CrossRef] [PubMed]

47. Bankevich, A.; Nurk, S.; Antipov, D.; Gurevich, A.A.; Dvorkin, M.; Kulikov, A.S.; Lesin, V.M.; Nikolenko, S.I.; Pham, S.; Prjibelski, A.D.; et al. SPAdes: A New Genome Assembly Algorithm and Its Applications to Single-Cell Sequencing. J. Comput. Biol. 2012, 19, 455-477. [CrossRef]

48. Seemann, T. Prokka: Rapid prokaryotic genome annotation. Bioinformatics 2014, 30, 2068-2069. [CrossRef]

49. Jolley, K.A.; Maiden, M.C.J. BIGSdb: Scalable analysis of bacterial genome variation at the population level. BMC Bioinform. 2010, 11, 595. [CrossRef]

50. Zhang, Z.; Schwartz, S.; Wagner, L.; Miller, W. A Greedy Algorithm for Aligning DNA Sequences. J. Comput. Biol. 2000, 7, 203-214. [CrossRef]

51. Zankari, E.; Hasman, H.; Cosentino, S.; Vestergaard, M.; Rasmussen, S.; Lund, O.; Aarestrup, F.M.; Larsen, M.V. Identification of acquired antimicrobial resistance genes. J. Antimicrob. Chemother. 2012, 67, 2640-2644. [CrossRef]

52. McArthur, A.G.; Waglechner, N.; Nizam, F.; Yan, A.; Azad, M.A.; Baylay, A.J.; Bhullar, K.; Canova, M.J.; De Pascale, G.; Ejim, L.; et al. The Comprehensive Antibiotic Resistance Database. Antimicrob. Agents Chemother. 2013, 57, 3348-3357. [CrossRef]

53. Chen, L.; Yang, J.; Yu, J.; Yao, Z.; Sun, L.; Shen, Y.; Jin, Q. VFDB: A reference database for bacterial virulence factors. Nucleic Acids Res. 2005, 33, D325-D328. [CrossRef]

54. Abramson, J.H. WINPEPI (PEPI-for-Windows): Computer programs for epidemiologists. Epidemiol. Perspect. Innov. 2004, 1, 6. [CrossRef]

Publisher's Note: MDPI stays neutral with regard to jurisdictional claims in published maps and institutional affiliations.

(C) 2020 by the authors. Licensee MDPI, Basel, Switzerland. This article is an open access article distributed under the terms and conditions of the Creative Commons Attribution (CC BY) license (http://creativecommons.org/licenses/by/4.0/). 